\title{
Variação de Abundância, Diversidade Ecológica e Similaridade de Coleoptera (Insecta) entre Restinga e Marisma do Estuário da Lagoa dos Patos, Rio Grande, RS
}

\author{
Katiele Dummel, Eduardo Alves Oliveira, Cristina Maria Loyola Zardo \& Fernando D'Incao
}

Universidade Federal do Rio Grande, e-mail: katieledummel@hotmail.com, eduardo.ao@gmail.com (Autor para correspondência ${ }^{\bowtie}$ ), dmbzardo@furg.br, docdinca@furg.br

EntomoBrasilis 4 (2): 39-44 (2011)

\begin{abstract}
Resumo. Insecta é o mais diverso, abundante e dominante grupo dos artrópodes, sendo, assim, utilizados em estudos de diversidade como indicadores biológicos. Contudo, poucos são os estudos a respeito da coleopterofauna e sua relação com os ambientes costeiros. As marismas e restingas se caracterizam por sua importância ecológica entre as regiões costeiras do Atlântico Sudoeste. Por isso, o presente trabalho teve por objetivo comparar a fauna de Coleoptera capturada em uma área de restinga com a fauna de uma marisma, no estuário da laguna Lagoa dos Patos, RS. As coletas foram realizadas no período de Agosto de 2007 à Julho de 2008 na área de restinga; e Setembro de 2008 à Agosto de 2009 na área de marisma. Ao total, foram coletados 826 coleópteros, distribuídos em 26 famílias. Os resultados apontam que tanto a restinga quanto a marisma apresentaram igual riqueza de famílias, embora com alternâncias de dominância entre as áreas. As flutuações das comunidades de coleoptera em cada área foram distintas ao longo do período, porém, os picos de abundância das duas áreas coincidiram com os meses mais quentes. A diversidade de famílias foi maior na área de restinga, sendo que as famílias capturadas na restinga possuem maior similaridade com aquelas encontradas em ambas as áreas. Grupos classificados como raros superaram os comuns. Famílias de hábito variado estiveram presentes nos dois ambientes; entretanto, os herbívoros dominaram na restinga e os carnívoros na marisma.
\end{abstract}

Palavras-chave: Coleoptera; estuário; marisma; restinga; similaridade.

\section{Changes in Abundance, Ecology Diversity and Similarity of Coleoptera (Insecta) Between Sandbank and salt Marsh of the Estuary of the Patos Lagoon, Rio Grande, RS}

Abstract. Insecta is the most diverse, abundant and dominant group of arthropods, being used in diversity studies as biological indicators. Despite this, there are few studies regard to coleopterofauna and its relation to coastal environments. Salt marshes and sandbanks are characterized by their ecological importance of coastal regions of the southwest Atlantic. Therefore, this study aimed to compare the fauna of Coleoptera captured in a sandbank area with the fauna of a salt marsh in the estuary of the lagoon Lagoa dos Patos, RS. Samples were collected from August 2007 to July 2008 in sandbank area; and from September 2008 to August 2009 in the salt marsh area. A total of 826 beetles were collected, distributed in 26 families. The results suggest that both sandbank and salt marsh presented equally rich families, but with alternation of dominance between the areas. Coleoptera communities flutuactions in each area were different in the period; however, the peak abundance of both areas coincided with the warmer months. The diversity of families was higher in the area of sandbank, while families caught in sandbanks have greater similarity to those found in both areas. Groups classified as rare surpassed common. Families of varied habit appeared in both environments; nonetheless, the herbivorous dominated in the sandbank and carnivorous in the salt marsh.

Keywords: Coleoptera; estuary; salt marsh; sandbank; similarity.

$\mathbf{O}$ s banhados e os habitats de águas profundas de diversos tipos caracterizam a planície costeira do extremo sul do Brasil, onde o complexo lagunar Patos-Mirim é a feição dominante. A Lagoa dos Patos e o oceano estão conectados através de um único canal, onde os eventos geomorfológicos regionais e a natureza deste caracterizam o sistema como uma Lagoa costeira do tipo "estrangulado", sendo classificada como a maior lagoa deste tipo no mundo (Asmus 1998). A área estuarina no sul da Lagoa é de $971 \mathrm{Km}^{2}$ (aproximadamente 10\% da Lagoa) (Asmus 1998), e pode ser destacada pela presença de ilhas com diferentes composições de flora, como as restingas e as marismas. A Lagoa dos Patos e seus entornos, particularmente o estuário e águas adjacentes, destacam-se entre as regiões costeiras do Atlântico Sudoeste pela sua importância ecológica (SEeliger et al. 1998), servindo de habitat para uma fauna muito diversa.

De acordo com Costa et al. (1997), os pisos mais elevados das marismas têm como residentes permanentes uma grande diversidade de invertebrados terrestres, particularmente os insetos. Axtell (1976); Dobson (1976); Cheng (1985) (apud
Williams \& Williams 1998) afirmam que a maioria dos insetos marinhos está associada a habitats costeiros, como estuários. Porém, insetos são declaradamente incomuns em habitats marinhos e, a partir de uma perspectiva de comparação interlaboratorial espacial/temporal, os estuários estão entre os menos estudados (Williams \& Hamm 2002). Em contra partida, estudos ecológicos e sobre o ciclo de vida dos animais aquáticos em regiões estuarinas vêm sendo desenvolvidos, por se tratar de áreas de grande fertilidade. Ainda assim, pouca atenção tem sido dada aos insetos nas avaliações ecológicas de habitat costeiro (AdAM 1990).

Insecta é o mais diverso, abundante e dominante grupo de artrópodes (TRIPLEHORn \& JOHNSON 2005), estando distribuídos em numerosas comunidades que compreendem cerca de $70 \%$ das espécies animais conhecidas. Os insetos tem sido alvo de estudos para que não apenas se conheça a sua diversidade, mas também sirvam de apoio para avaliação de condições ambientais [Southwood et al. 1979, Lawton 1983, Humphrey et al. 1999 (apud Ganho \& Marinoni 2003)], visto que grupos diversos de 
insetos são melhores usados como bioindicadores do que apenas espécies individuais de vertebrados (LANDREs et al. 1988, apud IANNUZZI et al. 2003). Os coleópteros representam o grupo mais rico dentre os insetos, com 350.000 espécies descritas (MACEDO et al. 2004), representando em torno de 40\% do total de espécies de Insecta, e 30\% de todas as espécies animais (LAWrence \& BRITTON 1991). Alguns grupos possuem boa parte das espécies com alta fidelidade ecológica, são altamente diversificados taxonômica e ecologicamente, facilmente coletáveis em grandes amostras e funcionalmente importantes nos ecossistemas (IANNuZzi et al. 2003).

O registro de besouros e outros invertebrados em zonas costeiras têm sido geralmente fragmentado (Foster 2000; LuXTON 2000, apud Eyre \& LuFF 2005). A distribuição de Coleoptera nas assembléias da costa nordeste da Inglaterra foi investigada por Eyre \& Luff (2005), e outros trabalhos sempre focalizaram um grupo específico de Coleoptera (Handke \& MENke 1995; Desender \& Maelfait 1999; Hammond 2000, apud Eyre \& Luff 2005). No Brasil, Dutra \& MiYAZAKi (1995) fizeram um levantamento dos insetos da Ilha do Mel, PR. MACEDO et al. (2004) inventariaram a fauna de besouros fitófagos da Restinga de Jurubatiba, RJ, e Oliveira et al. (2006) inventariaram a fauna de Insecta da Ilha dos Marinheiros, localizada no estuário da Lagoa do Patos.

Estudos de inventariamento são de fundamental importância quando se quer conhecer a fauna e definir a qualidade de vida dos organismos, bem como a avaliação do potencial de exploração dos recursos e de sua correta administração (GANHO \& MARINONI 2003). No entanto, o reconhecimento de tais organismos e mesmo o conhecimento mais amplo da riqueza global se inicia a partir de um processo sistematizado de inventariamento, com métodos de amostragem que possibilitem análises comparativas de dados (Oliveira 2006).

Devido ao escasso conhecimento sobre a fauna de Coleoptera em ambientes estuarinos da região sul do Brasil, colocou-se em prática dois projetos para se obter maiores informações acerca: o "Estudo da Diversidade da Entomofauna da Ilha do Leonídeo, Rio Grande, RS" e "Estrutura e Dinâmica da Assembléia de Insetos das Marismas do Estuário da Laguna Lagoa dos Patos, RS”. A partir dos dados obtidos nestes projetos, este trabalho teve por objetivo comparar a fauna de Coleoptera (Insecta) capturadas em áreas de restinga com a capturada em ambientes de marisma. Com este estudo, busca-se (a) conhecer a fauna e verificar a flutuação das comunidades de coleoptera nos diferentes ambientes estuarinos; (b) estimar a riqueza, diversidade e similaridade dos ambientes amostrados; (c) determinar grupos com maior freqüência e os raros para cada área; e (d) classificar as famílias quanto ao hábito alimentar.

\section{MATERIAL E MÉTODOS}

Área de Estudo. O estudo foi conduzido em duas ilhas do estuário da Lagoa dos Patos, Rio Grande, Rio Grande do Sul: a Ilha do Leonídeo e a Ilha da Torotama. A Ilha do Leonídeo (também conhecida como Ilha do Machadinho) está localizada no Saco do Justino, com limite sul a cidade do Rio Grande e ao norte a Ilha dos Marinheiros. A Ilha da Torotama está localizada entre o Saco do Silveira (limite norte) e o Saco do Arraial (limite sul) (Figura 1).

A área amostrada na Ilha do Leonídeo (Figura 1A) é uma área com típica cobertura vegetal de mata de restinga, avaliada, pela botânica Dra Lúcia Patrícia Dornelles do Instituto de Ciências Biológicas - FURG, como em bom estado de conservação. A área amostrada na Ilha da Torotama (Figura 1B) é uma área com cobertura de marismas intermediárias ou marismas esporadicamente alagadas (COSTA 1998), que são dominadas pela cobertura de Spartina densiflora Brong (macega).

Metodologia de coleta. Para a captura dos insetos foi utilizada uma armadilha de interceptação de vôo do tipo Malaise (ALMEIDA et al. 1998) em cada área. Na Ilha do Leonídeo as coletas foram realizadas durante o período de Agosto de 2007 à
Julho de 2008. Na Ilha da Torotama as coletas foram realizadas durante o período de Setembro de 2008 à Agosto de 2009. As amostras dos ambientes foram recolhidas mensalmente.
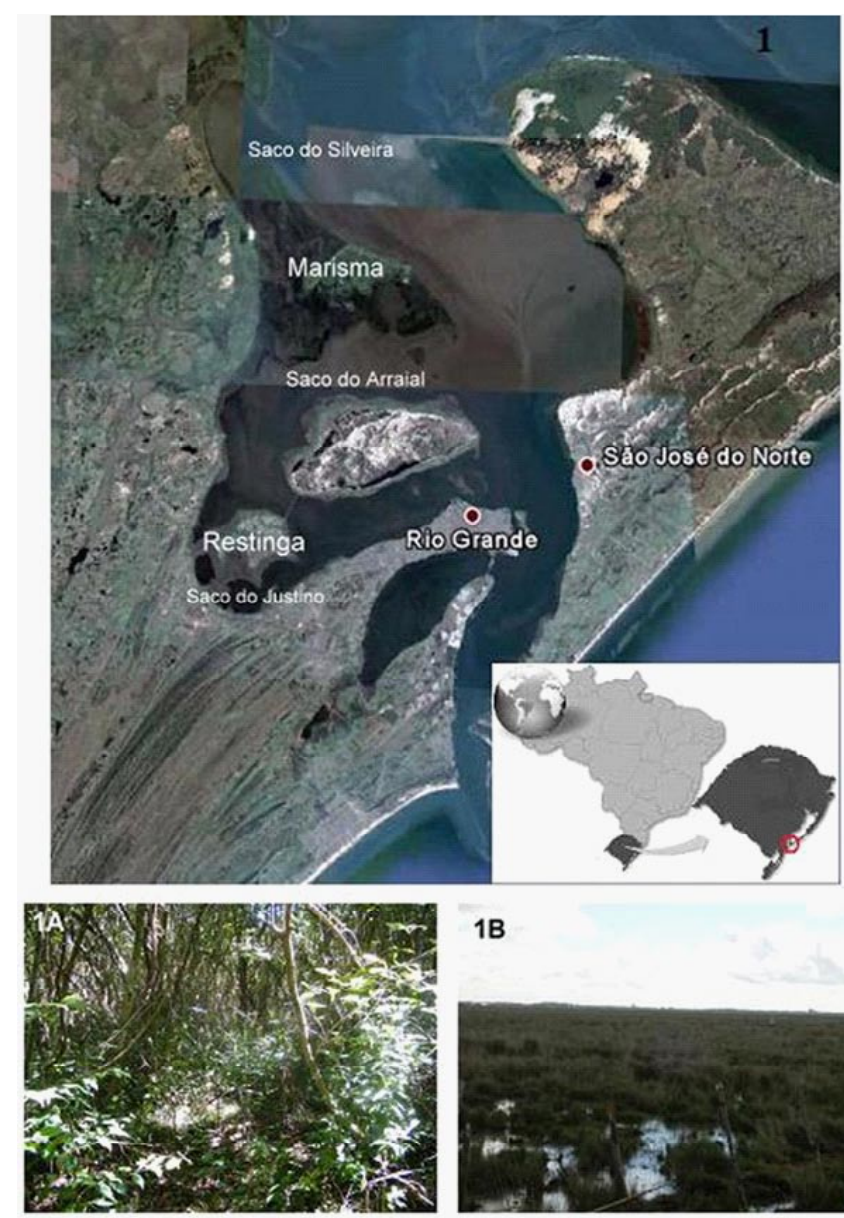

$1 \mathrm{~B}$

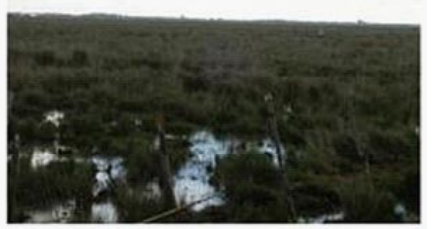

Figura 1. Imagem de satélite do Estuário da Lagoa dos Patos, Rio Grande, RS, com indicação dos pontos de coletas; $1 \mathrm{~A}$. Restinga da Ilha do Leonídeo; 1B. Marisma da Ilha da Torotama.

Triagem e Identificação. O material coletado foi depositado em recipientes, devidamente etiquetados, contendo álcool 70\%. A triagem dos coleópteros e das demais ordens capturadas na restinga foi realizada no Laboratório de Entomologia, do Instituto de Ciências Biológicas - ICB - FURG. Os coleópteros capturados nas marismas foram separados das demais ordens no Laboratório de Crustáceos Decápodes, do Instituto de Oceanografia - IO - FURG. As triagens ocorreram com auxílio de microscópios estereoscópios (20x).

Os exemplares foram identificados até o nível taxonômico de família (Triplehorn \& Johnson 2005). O material entomológico referente à área de restinga encontra-se depositado na Coleção Entomológica do ICB - FURG, enquanto que os espécimes capturados no ambiente de marisma foram depositados na Coleção Entomológica do IO - FURG.

Análise dos dados. Para tornar possíveis as análises estatísticas e de diversidade, os dados de captura do mês de Agosto/o7 da área de restinga foram deslocados para o mês de Agosto/o8. Assim, ficam equiparados os meses de início das coletas (Setembro) e fim (Agosto) para ambos os projetos, pois foram observadas poucas diferenças quanto às médias climáticas dos meses de Agosto de 2007 e 2008.

Através do teste de Shapiro-Wilk (W) testou-se a normalidade dos dados de abundância. Para verificar a existência de possíveis diferenças entre os locais amostrados, os mesmos dados de abundância mensal foram submetidos ao teste de Mann-Whitney para amostras independentes $(\mathrm{p}=0,05)$. As famílias foram classificadas quanto a sua abundância como "comuns" ( $>10$ indivíduos) e "raras" ( $\leq 10$ indivíduos) para cada área, seguindo a classificação proposta por ColwELl (2000). 
A fim de avaliar o estado da diversidade de Coleoptera e, com isso, estabelecer um parâmetro para o nível de conservação das marismas e das restingas locais, foi aplicado o índice de Diversidade e Dominância de Simpson (Rodriguts 2005). GANHO \& MARINONI (2005) aplicaram em seu estudo um valor de representatividade de $60 \%$ da coleopterofauna. Este parâmetro visa reconhecer as famílias que compõe, em abundância, a maior parcela das amostragens, sua posição de dominância frente às demais apoiada na análise de sua biologia, estimando como sendo este o reflexo do ambiente estudado. Este parâmetro de representatividade foi utilizado neste trabalho. As áreas amostradas foram comparadas através dos dados de presença/ ausência das famílias de Coleoptera, através do índice de Similaridade de Jaccard. Uma caracterização geral das áreas foi realizada através da análise dos hábitos alimentares das famílias de coleópteros, seguindo a classificação proposta por MARINONI et al. (2001).

\section{RESULTADOS}

Abundância e flutuação de Coleoptera. Neste estudo foram coletados 826 exemplares de coleópteros, representados por 26 diferentes famílias. A área de restinga apresentou a menor abundância, com 399 exemplares. Na área de marisma foram coletados 427 exemplares (Tabela 1). Na área de restinga, as cinco famílias mais abundantes representaram $87 \%$ do total amostrado (Chrysomelidae, Elateridae, Curculionidae, Ptilodactylidae e Cerambycidae). $\mathrm{Na}$ área de marisma as quatro famílias mais abundantes corresponderam $77 \%$ do total amostrado (Elateridae, Chrysomelidae, Curculionidae e Carabidae).

$\mathrm{Na}$ restinga os maiores pontos de flutuação da abundância ocorreram entre os meses de Setembro de 2007 e Janeiro de 2008, sendo os maiores picos registrados em Outubro de 2007 e Dezembro de 2007. De Janeiro a Agosto de 2008 a abundância dos besouros diminuiu, se mantendo sem alterações entre os meses de Março de 2008 e Agosto de 2008. (Figura 2A). Na marisma o maior ponto de flutuação ocorreu de Novembro de 2008 à Março de 2009, apresentando os maiores picos em Janeiro, Fevereiro e Março de 2009. De abril a agosto de 2009 a abundância diminui e se estabiliza entre Junho e Agosto de 2008 (Figura 2B).
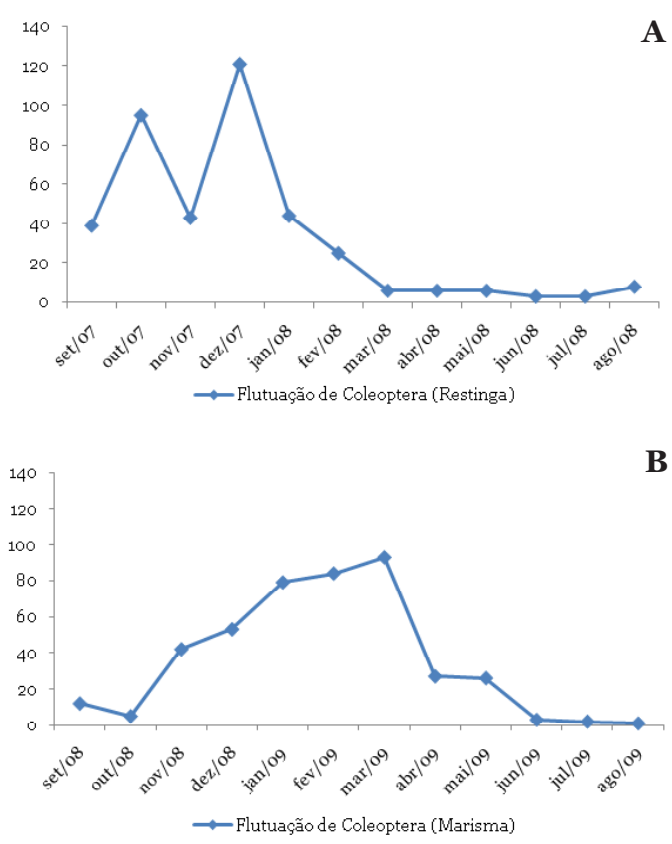

Figura 2. Flutuação mensal das famílias de Coleoptera capturadas por armadilha malaise em duas ilhas do estuário da Lagoa dos Patos, Rio Grande, RS. A. Restinga da Ilha do Leonídeo - período de Setembro de 2007 a Agosto de 2008; B. Marisma da Ilha da Torotama - período de Setembro de 2008 a Agosto de 2009.
Tabela 1. Grupo trófico, número de indivíduos e frequência absoluta por família de Coleoptera, capturados por armadilha Malaise de Agosto de 2007 até Julho de 2008 em uma restinga da Ilha do Leonídeo e, de Setembro de 2008 até Agosto de 2009 em uma marisma da Ilha da Torotama, estuário da Lagoa dos Patos, RS. (GT: Grupo Trófico. C: carnívoro; D: detritívoro; F: fungívoro; H: herbívoro.).

\begin{tabular}{lcccccc}
\hline \multicolumn{1}{c}{ Famílias } & GT & Restinga & \% & Marisma & \% & Total \\
\hline Anthicidae & C & 1 & 0,3 & 3 & 0,7 & 4 \\
\hline Bostrichidae & H & 3 & 0,8 & - & - & 3 \\
\hline Cantharidae & C & 1 & 0,3 & 12 & 2,8 & 13 \\
\hline Carabidae & C & 1 & - & 19 & 4,4 & 19 \\
\hline Cerambycidae & H & 33 & 8,3 & 8 & 1,9 & 41 \\
\hline Chrysomelidae & H & 110 & 27,6 & 105 & 24,6 & 215 \\
\hline Clambidae & F & - & - & 1 & 0,2 & 1 \\
\hline Cleridae & C & - & - & 3 & 0,7 & 3 \\
\hline Coccinellidae & C, H, F & 2 & 0,5 & 5 & 1,2 & 7 \\
\hline Cucujidae & H, C & 2 & 0,5 & - & - & 2 \\
\hline Curculionidae & F, H & 84 & 21,1 & 32 & 7,5 & 106 \\
\hline Elateridae & H, F, D & 88 & 22,1 & 176 & 41,2 & 264 \\
\hline Eucnemidae & H & 1 & 0,3 & - & - & 1 \\
\hline Lampyridae & C & - & - & 5 & 1,2 & 5 \\
\hline Lathridiidae & F & 4 & 1,0 & 14 & 3,3 & 18 \\
\hline Mordellidae & H & 11 & 2,8 & 1 & 0,2 & 12 \\
\hline Nitidulidae & D, F, & 3 & 0,8 & 17 & 4,0 & 20 \\
\hline Phalacridae & F, H & & & & & \\
\hline Ptilodactylidae & D & - & - & 2 & 0,5 & 2 \\
\hline Scarabaeidae & D, H & 16 & 4,0 & - & - & 16 \\
\hline Scirtidae & C & - & - & 6 & 1,4 & 6 \\
\hline Scydmaenidae & C & 2 & 0,5 & - & - & 2 \\
\hline Silphidae & D & 1 & 0,3 & - & - & 1 \\
\hline Staphylinidae & C, D, F & - & - & 17 & 4,0 & 17 \\
\hline Tenebrionidae & D, F & - & - & 1 & 0,2 & 1 \\
\hline Trogossitidae & C, F & 1 & 0,3 & - & - & 1 \\
\hline Total de indivíduos & & $\mathbf{3 9 9}$ & $\mathbf{1 0 0}$ & $\mathbf{4 2 7}$ & $\mathbf{1 0 0}$ & $\mathbf{8 2 6}$ \\
Total de famílias & & $\mathbf{1 9}$ & & $\mathbf{1 8}$ & & $\mathbf{2 6}$ \\
\hline & & & & & & \\
\hline
\end{tabular}

Riqueza, diversidade e similaridade de Coleoptera. Aárea de restinga registrou riqueza de 19 famílias, enquanto a área de marisma apresentou uma riqueza total de 18 famílias (Tabela 1). No total dos dois levantamentos, Chrysomelidae, Curculionidae e Elateridae representaram as maiores dominâncias de coleópteros coletados. Clambidae, Eucnemidae, Silphidae, Tenebrionidae e Trogossitidae tiveram apenas um representante durante todo o período do levantamento.

Do total de famílias capturadas, 12 foram classificadas como comuns e as demais 14 como raras. A área de marisma apresentou maior número de famílias comuns (08) quando comparado à restinga (07). Chrysomelidae, Curculionidae e Elateridae foram as únicas consideradas comuns à ambas áreas amostradas. Já Cerambycidae, Mordellidae, Ptilodactylidae e Scarabaeidae foram comuns apenas na restinga, enquanto Cantharidae, Carabidae, Lathridiidae, Nitidulidae e Staphylinidae foram comuns para a marisma. Anthicidae e Coccinellidae foram consideradas raras nas duas áreas. A restinga e a marisma abrigaram, cada uma, seis famílias raras e exclusivas. De todo material amostrado, onze famílias foram pertinentes às duas áreas: Anthicidae, Cantharidae, Carabidae, Cerambycidae, Chrysomelidae, Coccinellidae, Curculionidae, Elateridae, Lathridiidae, Mordelidae e Nitidulidae. Na área de restinga oito famílias foram exclusivas: Bostrichidae, Cucujidae, Eucnemidae, Ptilodactylidae, Scarabaeidae, Silphidae, Scydmaenidae e Trogossitidae. Na área de marisma sete famílias foram exclusivas: Cleridae, Lampyridae, Phalacridae, Scirtidae, Staphylinidae e Tenebrionidae. 
Foi verificada a anormalidade dos dados de abundância $(\mathrm{W}=0,78 ; \mathrm{p}=0,005) . \mathrm{O}$ teste de Mann-Whitney aceitou a hipótese nula $(U=357 ; p=0,56)$, indicando que não há diferenças significativas entre as áreas.

Através do cálculo do índice de Diversidade e Dominância de Simpson, afirma-se que ambas as áreas possuem alta diversidade de coleópteros (Ds = 0,89) e baixa dominância (l $=0,10)$. A área de restinga mostrou-se mais diversa $(\mathrm{Ds}=0,81)$ do que a área de marisma (Ds=0,75). O índice de dominância evidenciou maior dominância na área de marisma $(1=0,24)$ do que na área de restinga $(l=0,18)$.

A partir de uma matriz de presença/ausência, o dendrograma de similaridade de Jaccard demonstrou maior relacionamento das famílias exclusivas da área de restinga com as famílias presentes em ambas as áreas (Coef. Correl. Cofenética $=0,88$ ) (Figura 3).

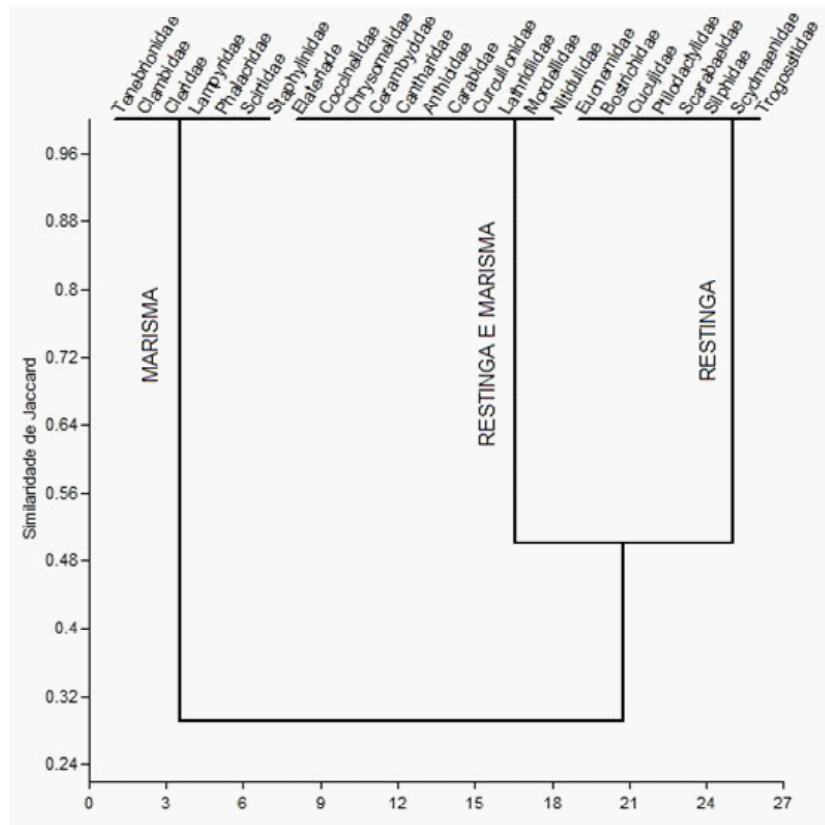

Figura 3. Dendograma de similaridade de Jaccard, confeccionado a partir dos dados de presença/ausência na captura de famílias de Coleoptera por armadilha malaise, em duas ilhas com diferentes coberturas vegetais no estuário da Lagoa dos Patos, Rio Grande, RS, durante os períodos de Agosto de 2007 a Julho de 2008 e Setembro de 2008 a Agosto de 2009.

Grupos Tróficos. Em meio as 26 famílias coletadas, dez possuem hábitos alimentares variados (com representantes herbívoros, detritívoros, carnívoros e fungívoros), sete exclusivamente de hábito carnívoro, cinco herbívoro, dois detritívoro e dois fungívoro (Tabela 1). No ambiente de restinga ocorreram famílias de todos os hábitos alimentares, sendo sete famílias de hábitos variados, cinco famílias de hábito herbívoro, quatro famílias de hábito carnívoro, duas de hábito detritívoro e uma família de hábito fungívoro. Na marisma sete famílias são de hábitos variados, seis de hábito carnívoro, três herbívoros e duas de hábito fungívoro. Nesta área não foram capturadas famílias de hábito exclusivamente detritívoro.

\section{DISCUSSÃO}

Dutra \& Miyazaki (1995) durante seu estudo na área Fortaleza, localidade da Ilha do Mel, a $400 \mathrm{~m}$ da zona de maré, capturaram 1083 coleópteros. Segundo comparação florística, o ambiente da localidade Fortaleza constitui-se de uma área de restinga, cuja formação vegetal é a mais próxima ao ambiente de restinga do presente trabalho. Oliveira (2006) durante seu inventariamento em uma restinga em semelhante estado de conservação na Ilha dos Marinheiros, localizada no estuário da Lagoa dos Patos, capturou 386 coleópteros, distribuídos em 18 famílias. Na restinga da Ilha do Leonídeo a abundância total de indivíduos apresentou um número visivelmente menor do que o encontrado por Dutra \& MiYAZAKI (1995), porém, muito próximo ao número de coleópteros encontrados por Oliveira (2006). A proximidade entre as abundâncias do presente trabalho e o de Oliveira (2006) pode indicar que os ambientes de restinga das ilhas do estuário da Lagoa dos Patos possuem abundâncias de Coleoptera semelhantes. A diferença de abundância entre as restingas estuarinas da Lagoa dos Patos e o ambiente da Fortaleza na Ilha do Mel pode ser indicativa dos efeitos latitudinais sobre os pontos de coleta, influência da arquitetura vegetal (HUTCHESON 1990), condições do solo no desenvolvimento de larvas e eventos climatológicos isolados (i. e. falta ou excesso de chuva). Tais resultados possibilitam estimar um padrão de abundância de Coleoptera para as restingas da Lagoa dos Patos. A proximidade dos totais de abundância entre restinga e marisma neste estuário pode permitir que o padrão visto na restinga sirva como referência para uma estimativa de captura nas marismas.

As cinco famílias dominantes do estudo de Dutra \& MiYAZAKI (1995) na Fortaleza, representaram cerca de 60\% do total amostrado, sendo elas Scirtidae, Mordelidae, Chrysomelidae, Phengodidae e Curculionidae, em ordem decrescente de abundância. As cinco famílias dominantes do estudo de OLIVEIRA (2006) representaram 64\% do total, sendo elas Cerambycidae, Curculionidae, Scirtidae, Nitidulidae e Elateridae, em ordem decrescente de abundância. As mesmas cinco famílias dominantes da restinga da Ilha do Leonídeo representaram apenas $27 \%$ do total capturado na Fortaleza, e 56\% do total capturado na Ilha dos Marinheiros. A partir disto acredita-se que a abundancia das famílias difere entre as diversas formações de restingas do litoral sul brasileiro, com exceção de Chrysomelidae, Curculionidae e Elateridae, que se mostram entre as famílias mais abundantes nos três estudos acima citados.

Além da semelhança na abundância entre restinga e marisma, verifica-se que as famílias de maior representatividade são parcialmente as mesmas para ambas as áreas. Isto pôde ser afirmado através dos testes de homogeneidade das variâncias. Embora a arquitetura vegetal que constitui os dois locais de coleta seja muito diferente, outros fatores podem contribuir para tal resultado. Diferenças de concentração da salinidade pela distância da margem da laguna, o teor de umidade retido pelo solo e a proximidade natural existente entre restingas e marismas são pontos a serem considerados como influentes nos resultados de abundância das comunidades de Coleoptera estuarinas.

Os Chrysomelidae e Elateridae foram as famílias mais abundantes neste estudo, porém ocorreu uma inversão de dominância nas duas áreas (restinga e marisma), consistindo Chrysomelidae como a mais abundante na restinga, e Elateridae a mais abundante na marisma. Os números de crisomelídeos não apresentaram uma discrepância elevada como visto para os elaterídeos entre as duas áreas. Tal situação pode estar atrelada ao fato das larvas de Elateridae viverem em solos barrosos ou arenosos onde se alimentam de raízes (MARINoni et al. 2001), padrão encontrado nas marismas.

Quando comparados, os maiores e os menores pontos de flutuação da abundância entre a restinga e a marisma, é possível inferir que algumas famílias utilizam a restinga nos meses típicos de primavera e verão para sua reprodução (oviposição, desenvolvimento larval, metamorfose e emergência do adulto), alimentação ou proteção de intempéries climáticas. As comunidades de Coleoptera podem exaurir os recursos desta área, elevando a competição inter ou intraespecíficas, o que leva a procura de novos locais para dispersão, neste caso, a marisma, nos meses de verão. Tais padrões temporais ainda indicam que as famílias presentes nas duas áreas apresentem uma só estação reprodutiva por ano, com picos nos meses mais quentes.

Os índices de diversidade e dominância estiveram muito próximos nas duas áreas estudadas, ambas com alto índice de diversidade e baixo índice de dominância. $\mathrm{O}$ alto valor da diversidade aliado a baixa dominância encontrado demonstra que tanto o ambiente de restinga quanto o ambiente de marisma apresentam-se estáveis, não suportando o favorecimento à 
famílias de coleópteros em específico.

$\mathrm{O}$ alto grau de diversidade da restinga da Ilha do Leonídeo está relacionado com a presença de micro-habitats e com a salinidade do ambiente. $\mathrm{O}$ valor de dominância mais elevado na marisma pode estar aliado às condições químicas do ambiente; o estuário apresenta variações diárias e até horárias de constituintes químicos, como os níveis de salinidade (NIENCHESKI \& Baumgarten 1998). Assim, o ambiente de marisma, que está periodicamente sendo alagado pelas águas da laguna, estaria mais favorável às famílias mais bem adaptadas a determinadas condições, impostas por esta proximidade com a laguna. De fato, no dendograma foi verificada maior similaridade entre as famílias exclusivas da área de restinga com as famílias presentes em ambas as áreas (restinga e marisma), e um distanciamento das famílias exclusivas da área de marisma. Tal relação reforça a hipótese de que as famílias exclusivas das marismas apresentam mais recursos alimentares e adaptações fisiológicas para ocuparem estes ambientes. Supõem-se ainda que as famílias comuns a ambas as áreas podem se locomover de uma área para outra, sendo dependentes das condições climáticas e químicas ao longo do período de estudo.

A dominância de determinados grupos, como Chrysomelidae, Curculionidae e Elateridae deve-se ao fato de que representam as mais bem sucedidas famílias da ordem, apresentando grande variabilidade de formas e ampla distribuição geográfica (LAWREnce \& BRITTON 1991). Chrysomelidae é um grupo muito variado quanto ao aspecto e hábito (TrIPLEHORN \& Johnson 2005). Curculionidae são os coleópteros mais ricos em espécies, apresentando uma grande variedade de hábitos (ARAUJO et al. 2009). Elateridae representa um grande grupo da ordem Coleoptera, tendo várias espécies muito comuns (BorRoR \& DELONG 1969).

A presença de famílias de hábitos variados, tanto na restinga quanto na marisma, indica que existe uma variabilidade de alimentos disponíveis para as famílias da ordem Coleoptera no ambiente estuarino. O alto índice de herbívoros na área de restinga indica que há uma disponibilidade e variabilidade alta de espécies vegetais para os coleópteros. Entre os herbívoros, quatro famílias foram comuns às duas áreas do estudo: Cerambycidae, Chrysomelidae, Curculionidae e Mordelidae, sendo que, as quatro famílias apresentaram maior abundância na restinga.

Nas marismas, famílias carnívoras e fungívoras verificou-se a maior presença. Infere-se que há grande disponibilidade não só de pequenos insetos, mas também de aracnídeos e moluscos para os coleópteros predadores. Longos períodos de encharcamento e alta umidade concentrada entre os talos da vegetação de Spartina alterniflora Loisel) favorecem o crescimento de fungos e proporciona a ocupação dos coleópteros fungívoros. A biomassa de S. alterniflora que entra em decomposição no solo é rapidamente consumida pelos besouros detritívoros Tenebrionidae e Staphylinidae. Duas famílias de hábito exclusivamente carnívoro (Anthicidae e Cantharidae) foram capturadas em ambas as áreas, entretanto, a abundância destas se manteve maior na área de marisma.

A maioria das famílias consideradas comuns está entre os coleópteros que apresentam o maior número de espécies, como Carabidae, Cerambycidae, Chrysomelidae, Curculionidae, Elateridae, Scarabaeidae e Staphylinidae (IANuzzi et al. 2003). As demais famílias comuns podem estar correspondendo às adaptações que possuem para os ambientes estudados.

Os ambientes de ilha do estuário da Lagoa dos Patos apresentam riqueza significativa de famílias de Coleoptera, o que pode ser um parâmetro indicativo de conservação ambiental do ambiente estuarino. A restinga e a marisma, apesar de apresentarem diferentes arquiteturas vegetais, demonstram abundâncias semelhantes. Ambos ambientes possuem famílias em comum e exclusivas, em um número muito próximo. A presença de todos os hábitos tróficos nestes ambientes indica que há disponibilidade de alimento para uma variada fauna de Coleoptera.

\section{REFERÊNCIAS}

Adam, P., 1990. Saltmarsh Ecology. Cambridge, Cambridge University Press, 461p.

Almeida, L.M., C.S. Ribeiro-Costa \& L. Marinoni, 1998. Manual de Coleta, Conservação, Montagem e Identificação de Insetos. Ribeirão Preto, Editora Holos, 78p.

Araujo, C.O., V. Flinte, M.V. Macêdo \& R.F. Monteiro, 2009. Ecologia e variação espacial de Naupactus lar Germar (Coleoptera, Curculionidae, Entiminae) no Parque Nacional da Restinga de Jurubatiba, RJ. Revista Brasileira de Entomologia, 53: 82-87.

Asmus, M.L., 1998. A planície costeira e a Lagoa dos Patos, p 9-12. In: U. Seeliger; C. Odebrecht \& J.P. Castello (Eds), Os Ecossistemas Costeiro e Marinho do Extremo Sul do Brasil. Ecoscientia, Rio Grande, 341p.

Colwell, R.K., 2000. EstimateS: Statistical estimation of species richness and shared species from samples. Version 5. User's Guide Draft 7 March 2001. Disponível em: $<$ http://viceroy.eeb.uconn.edu/estimates > [20/10/2009].

Costa, C.S.B., 1998. Plantas de Marismas e Terras Alagáveis, p 25-29. In: U. Seeliger; C. Odebrecht \& J.P. Castello (eds), Os Ecossistemas Costeiro e Marinho do Extremo Sul do Brasil. Ecoscientia, Rio Grande, 341p.

Costa, C.S.B., U. Seeliger, C.P.L. Oliveira \& A.M.M. Mazo, 1997. Distribuição, funções e valores das marismas e pradarias submersas no estuário da Lagoa dos Patos (RS, BRASIL). Atlântica, Rio Grande, 19: 67-85.

Dutra, R.R.C. \& R.D. Miyazaki, 1995. Famílias de Coleoptera capturadas com armadilha malaise em duas localidades da Ilha do Mel, Baía de Paranaguá, Paraná, Brasil. Arquivos de Biologia e Tecnologia, 38: 175-190

Eyre, M.D. \& M.L. Luff, 2005. The Distribution of Epigeal Beetle (Coleoptera) assemblages on the north-east England Coast. Journal of Coastal Research, 21: 982-990.

Freire, M.S.B., 1990. Levantamento florístico do Parque Estadual das Dunas de Natal. Acta Botanica Brasilica, 4: 41-59.

Ganho, N.G. \& R.C. Marinoni, 2003. Fauna de Coleoptera no Parque de Vila Velha, Ponta Grossa, Paraná, Brasil. Abundância e riqueza das famílias capturadas através de armadilhas malaise. Revista Brasileira de Zoologia, 20: 727736.

Ganho, N.G. \& R.C. Marinoni, 2005. A diversidade inventarial de Coleoptera (Insecta) em uma paisagem antropizada do Bioma Araucária. Revista Brasileira de Entomologia. 49: 535-543.

Hutcheson, J., 1990. Characterization of terrestrial insect communities using quantified, Malaise-trapped Coleoptera. Ecological Entomology, 15: 143-151.

Iannuzzi, L., A.C.D. Maia, C.E.B. Nobre, D.K. Suzuki \& F.J.A. Muniz, 2003. Padrões locais de diversidade de Coleoptera (Insecta) em vegetação de Caatinga, p. 367-389. In: Leal, I.R., M. Tabarelli \& J.M.C. Silva. (Org.). Ecologia e conservação da caatinga. Editora Universitária da Universidade Federal do Pernambuco, Recife, 822p.

Lawrence, J.F. \& E.B. Britton, 1991. Coleoptera. p. 543-683 In: The Insects of Australia. Vol. 2. Melbourne University Press., Australia, 744p.

Macedo, M.V., V. Grenha, V. Flinte \& T. S. Rabello, 2004. Besouro Fitófagos da Restinga de Jurubatiba, p. 117-126. In: Rocha, C.F.D., F.A. Esteves \& F.R. Scarano (Eds.). Pesquisas de longa duração Restinga de Jurubatiba: ecologia, história natural e conservação. RIMA. São Carlos, SP, 376p.

Marinoni, R.C., N.G. Ganho, M.L. Monné, \& J.R.M. Mermudes, 2001. Hábitos alimentares em Coleoptera (Insecta). Ribeirão Preto, Editora Holos, 63p.

Neincheski, L.F. \& M.G. Baumgarten, 1998. Química Ambiental, p. 21-25. In: U. Seeliger; C. Odebrecht \& J.P. Castello (eds), Os Ecossistemas Costeiro e Marinho do Extremo Sul do Brasil. Ecoscientia, Rio Grande, 341p.

Oliveira, E.A., L.V.Nascimento \& C.M.L.Zardo, 2006. Abundância e padrão sazonal da entomofauna de restinga em uma ilha do 
estuário da laguna Lagoa dos Patos, Rio Grande, RS, Brasil. Estudos em Biologia, 28: 27-35.

Oliveira, E.A., 2006. Coleópteros de uma ilha estuarina da Lagoa dos Patos, Rio Grande, Rio Grande do Sul, Brasil. Dissertação de Mestrado. Universidade Federal do Paraná. Disponível em: $<$ http://dspace.c3sl.ufpr.br/dspace/handle/1884/4723>. [15/08/2009].

Reitz, R., 1961. Vegetação da zona marítima de Santa Catarina. Sellowia, 13: 17-111.

Rodrigues, W.C., 2005. DivEs - Diversidade de espécies. Versão 2.o. Software e Guia do Usuário. Disponível em: <http://www.ebras.bio.br/dives $>$ [15/10/2008].

Seeliger, U., C.S.B. Costa \& P.C. Abreu, 1998. Os Ecossistemas Costeiros e Marinhos do Extremo Sul do Brasil (Seeliger, U., C. Odebrecht \& J.P. Castello. Eds.). Ecoscientia. Rio Grande. 341p.

Statsoft Inc., 2007. STATISTICA (data analysis software system), version 8.o. Disponível em: www.statsoft.com [15/09/2009].

\section{Como citar este artigo:}

Dummel, K., E.A. Oliveira, C.M.L. Zardo \& F. D’Incao, 2011. Variação de Abundância, Diversidade Ecológica e Similaridade de Coleoptera (Insecta) entre Restinga e Marisma do Estuário da Lagoa dos Patos, Rio Grande, RS. EntomoBrasilis, 4(2): 39-44. www.periodico.ebras.bio.br/ojs
Triplehorn, C.A \& N.F Johnson, 2005. Borror \& Delong's Introduction to the Study of insects. Thomsom Brooks/Cole. 653p.

Williams, D.D. \& N.E. Williams, 1998. Aquatic insects in an estuarine environment: densities, distribution and salinity tolerance. Freshwater Biology, 39: 411-421.

Willians, D.D. \& T. Hamm, 2002. Insect community organisation in estuaries: the role of the physical environment. Ecography, 25: 372-384.

Recebido em: 11/06/2010

Aceito em: 12/11/2010
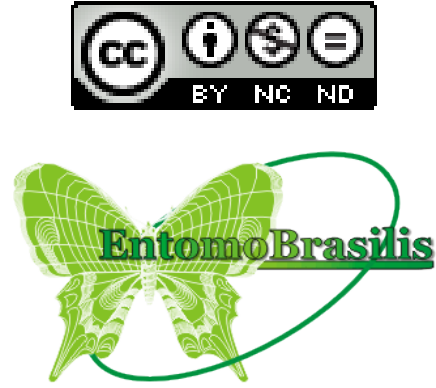